\title{
Antibodies Against Egg- and Cell-Grown Influenza A(H3N2) Viruses in Adults Hospitalized During the 2017-2018 Influenza Season
}

\author{
Min Z. Levine, ${ }^{1, a}$ Emily T. Martin, ${ }^{2, a}$ Joshua G. Petrie, ${ }^{2}$ Adam S. Lauring, ${ }^{3}$ Crystal Holiday, ${ }^{1}$ Stacie Jefferson, ${ }^{1}$ William J. Fitzsimmons, ${ }^{3}$ Emileigh Johnson, ${ }^{2}$ \\ Jill M. Ferdinands, ${ }^{1}$ and Arnold S. Monto ${ }^{2}$ \\ ${ }^{1}$ Influenza Division, Centers for Disease Control and Prevention, Atlanta, Georgia; and ${ }^{2}$ Department of Epidemiology, University of Michigan School of Public Health, and ${ }^{3}$ Division of Infectious \\ Diseases, Department of Internal Medicine, University of Michigan, Ann Arbor, Michigan
}

Background. Influenza vaccine effectiveness was low in 2017-2018, yet circulating influenza A(H3N2) viruses were antigenically similar to cell-grown vaccine strains. Notably, most influenza vaccines are egg propagated.

Methods. Serum specimens were collected shortly after illness onset from 15 influenza A(H3N2) virus-infected cases and 15 uninfected hospitalized adults. Geometric mean titers against egg- and cell-grown influenza A/Hong Kong/4801/2014(H3N2) virus vaccine strains and representative circulating viruses (including A/Washington/16/2017) were determined by a microneutralization $(\mathrm{MN})$ assay. Independent effects of strain-specific titers on susceptibility were estimated by logistic regression.

Results. MN titers against egg-grown influenza A/Hong Kong virus were significantly higher among vaccinated individuals (173 vs $41 ; P=0.01$ ). In unadjusted models, a 2 -fold increase in titers against egg-grown influenza $\mathrm{A} / \mathrm{Hong}$ Kong virus was not significantly protective ( $29 \%$ reduction; $P=.09$ ), but a similar increase in the cell-grown influenza $\mathrm{A} /$ Washington virus antibody titer (3C.2a2) was protective ( $60 \%$ reduction; $P=.02$ ). Higher egg-grown influenza $\mathrm{A} /$ Hong Kong virus titers were not significantly associated with infection, when adjusted for antibody titers against influenza A/Washington virus (15\% reduction; $P=.61$ ). A $54 \%$ reduction in the odds of infection was observed with a 2 -fold increase in titer against influenza $\mathrm{A} /$ Washington virus ( $P=$ not significant), adjusted for the titer against egg-grown influenza A/Hong Kong virus titer.

Conclusion. Individuals vaccinated in 2017-2018 had high antibody titers against the egg-adapted vaccine strain and lower titers against circulating viruses. Titers against circulating but not egg-adapted strains were correlated with protection.

Keywords. Influenza A; antibodies; egg adaptation; vaccine effectiveness.

The 2017-2018 influenza season was severe in the United States, resulting in the highest rate of influenza-related hospitalizations since the Centers for Disease Control and Prevention (CDC) began including adults in inpatient surveillance [1]. Although the influenza $\mathrm{A}(\mathrm{H} 3 \mathrm{~N} 2)$ viruses that circulated in the United States belonged to multiple genetic groups, they were all considered antigenically similar to cell-grown vaccine-strain reference viruses [2]. However, the majority of available influenza vaccines are produced in eggs, and vaccine effectiveness (VE) was low against adult influenza $\mathrm{A}(\mathrm{H} 3 \mathrm{~N} 2)$ virus infections in 2017-2018 [3]. While the inadequate VE against influenza $\mathrm{A}(\mathrm{H} 3 \mathrm{~N} 2)$ virus infection was highlighted by the intensity of the 2017-2018 outbreak, the national level estimates

Received 11 October 2018; editorial decision 23 January 2019; accepted 29 January 2019; published online February 4, 2019

${ }^{a}$ M. Z .L. and E. T. M. contributed equally to this work.

Correspondence: E. T. Martin, PhD, Department of Epidemiology, University of Michigan School of Public Health, Ann Arbor, MI (etmartin@umich.edu).

The Journal of Infectious Diseases ${ }^{\circledR} \quad$ 2019;219:1904-12

(C) The Author(s) 2019. Published by Oxford University Press for the Infectious Diseases Society of America. All rights reserved. For permissions, e-mail: journals.permissions@oup.com. DOI: 10.1093/infdis/jiz049 of the VE against influenza $\mathrm{A}(\mathrm{H} 3 \mathrm{~N} 2)$ virus were consistent with observations of a relatively low VE against this subtype throughout the past decade [4].

Studies have suggested that changes required to adapt influenza viruses for growth in eggs in still widely used egg-based vaccines may have contributed to low VE in years with influenza A(H3N2) virus circulation, including 2012-2013, 2016-2017, and 2017-2018 [5-7]. Egg-related changes can happen in multiple hemagglutinin (HA) epitopes, and those egg-adaptation sites of concern have changed since the 2014-2015 season. Specifically, influenza $\mathrm{A}(\mathrm{H} 3 \mathrm{~N} 2)$ strains belonging to the $3 \mathrm{C} .2 \mathrm{a}$ genetic group, which emerged in 2014-2015, acquired a K160T mutation in the gene encoding HA, leading to the addition of a glycosylation site at $\mathrm{HA}$ amino acid position 158 in antigenic site B [6-8]. Glycans can shield and redirect HA epitopes. During the vaccine production process, vaccine virus adaptation to eggs can cause a T160K reversion and the loss of glycosylation, altering the antigenicity of the egg-based vaccines [7]. Furthermore, additional egg-adapted changes, including HA substitutions such as the L194P change in antigenic site B that is often present in egg-adapted influenza $\mathrm{A}(\mathrm{H} 3 \mathrm{~N} 2)$ virus, can also affect vaccine antigenicity [9]. As a result, human sera collected following 
vaccination with egg-based influenza $\mathrm{A}(\mathrm{H} 3 \mathrm{~N} 2)$ vaccines have shown reduced inhibition against recently circulating influenza $\mathrm{A}(\mathrm{H} 3 \mathrm{~N} 2)$ virus $[7,2]$. The objective of this study was to compare antibody titers against both circulating influenza $\mathrm{A}(\mathrm{H} 3 \mathrm{~N} 2)$ strains and egg-adapted influenza $\mathrm{A}(\mathrm{H} 3 \mathrm{~N} 2)$ strains in acute specimens from individuals with and those without influenza virus infection in the 2017-2018 season.

\section{METHODS}

During the severe 2017-2018 influenza season, we performed a study of the effectiveness of influenza vaccine among inpatients with acute respiratory illness as part of the CDC-sponsored Hospitalized Adult Influenza Vaccine Effectiveness Network (HAIVEN). At the HAIVEN site in Michigan, serum specimens were collected shortly after illness onset analyzed to determine the preinfection serologic immune status [10].

\section{Study Population}

Adult patients (age, $\geq 18$ years) hospitalized for treatment of acute respiratory illnesses at the University of Michigan Hospital in Ann Arbor were prospectively enrolled in a case test-negative design study of the effectiveness of influenza vaccine during the 2017-2018 influenza season, using previously described methods [11]. All patients were enrolled $\leq 10$ days after illness onset during active influenza virus circulation (Figure 1). Participants provided informed consent, completed an enrollment interview, and had throat and nasal swab specimens collected for influenza virus identification by reverse transcription polymerase chain reaction (RT-PCR) analysis, using the CDC Influenza Division protocol. As part of the enrollment interview, participants rated their general health prior to the onset of their current illness (options were "excellent," "very good,"

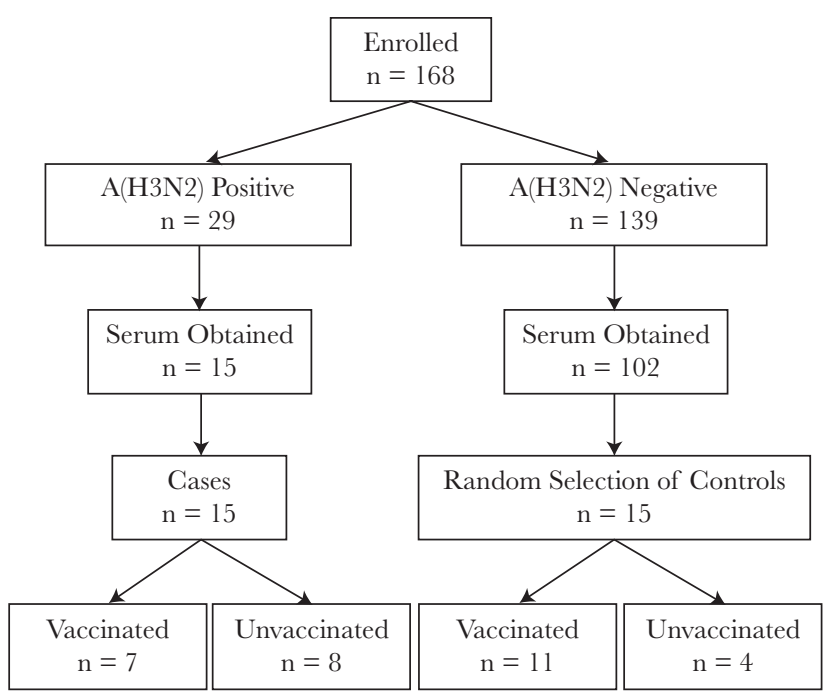

Figure 1. Consolidation Standard of Reporting Trials diagram showing enrollment and sample availability for 168 individuals enrolled in the Hospitalized Adult Influenza Vaccine Effectiveness Network study at the time of testing. "good," "fair," and "poor") and, to define frailty status, responded to a series of 5 questions assessing difficulties with various tasks [11-13]. General health was treated as a dichotomous variable ( $\geq$ good vs $\leq$ fair), and participants were considered frail if they reported difficulties in $\geq 3$ of the 5 elements of the frailty interview. Influenza vaccination status was documented by electronic medical record and review of the state vaccination registry.

The institutional review boards at the University of Michigan Medical School and Centers for Disease Control and Prevention reviewed and approved the study.

\section{Serologic Analysis}

Available residual clinical serum specimens collected after hospital admission (ie, 0-9 days after illness onset) were retrieved. Specimens were considered to represent preinfection serologic status, prior to a de novo antibody response to the incident illness. Among participants enrolled as of 31 January 2018 with available serum specimens, we selected all 15 influenza A(H3N2) virus-positive cases identified to date and 15 influenza virusnegative controls matched (by nearest neighbor matching) on age and date of enrollment (Figure 1). Microneutralization MN assays were performed using Madin-Darby canine kidney (MDCK)-SIAT1 cells [14, 15]. In brief, sera were inactivated by heat, subjected to 2 -fold serial dilution, mixed with $10050 \%$ tissue culture infective doses of influenza $\mathrm{A}(\mathrm{H} 3 \mathrm{~N} 2)$ viruses and incubated at $37^{\circ} \mathrm{C}$ with $5 \% \mathrm{CO}_{2}$ for 1 hour. The virus-sera mixture was used to infect $1.5 \times 10^{4} \mathrm{MDCK}-\mathrm{SIAT} 1$ cells/well, and incubation was performed for $18-20$ hours at $37^{\circ} \mathrm{C}$ with $5 \% \mathrm{CO}_{2}$. After cold acetone fixation, the presence of viral protein was quantified by an enzyme-linked immunosorbent assay, using monoclonal antibodies specific to the nucleoproteins of the influenza A viruses. $\mathrm{MN}$ assays were conducted in the Influenza Division research laboratory at the CDC. MN antibody titers were measured against influenza $\mathrm{A}(\mathrm{H} 3 \mathrm{~N} 2)$ viruses representing the egg- and cell culture-grown influenza A/Hong Kong/4801/2014 virus included in 2017-2018 northern hemisphere influenza vaccines and 4 wild-type viruses representative of circulating influenza $\mathrm{A}(\mathrm{H} 3 \mathrm{~N} 2)$ virus genetic groups (Table 1).

Neuraminidase-inhibition (NAI) antibody titers were determined for all 30 serum specimens collected from case and control participants using enzyme-linked lectin assays in the Respiratory Virus Laboratory at the University of Michigan School of Public Health [16]. NAI titers were measured using $\mathrm{H} 6$ reassortant influenza viruses (kindly provided by $M$. Eichelberger and $H$. Wan) bearing neuraminidase representing the influenza A/Hong Kong/4801/2014(H3N2) vaccine in the 2017-2018 season and influenza A/Singapore/ INFIMH-16-0019/2016(H3N2) strains.

Antibody titers were calculated as the reciprocal of the highest dilution that neutralized $50 \%$ of virus infectivity (based on $\mathrm{MN}$ assays) or inhibited neuraminidase activity (based on 


\begin{tabular}{|c|c|c|c|}
\hline Strain & Propagation & Genetic Group & $\begin{array}{l}\text { Viruses Identified in } \\
\text { Study Surveillance, \% }\end{array}$ \\
\hline A/HongKong/4801/2014 & Egg & 3 C. $2 a$ & 0 \\
\hline A/HongKong/4801/2014 b & MDCK-SIAT1 cells & $3 C .2 a$ & 0 \\
\hline AMashington/16/2017 & MDCK-SIAT1 cells & 3 C. $2 \mathrm{a} 2$ & 83 \\
\hline A/Singapore/Infimh-16-0019/2016 & MDCK-SIAT1 cells & $3 C .2 \mathrm{a} 1$ & 4 \\
\hline A/Maryland/53/2017 & MDCK-SIAT1 cells & $3 C \cdot 2 a 1 b+135 N$ & 0 \\
\hline AMisconsin/327/2017 & MDCK-SIAT1 cells & $3 C .2 a 1 b+135 K$ & 0 \\
\hline
\end{tabular}

Abbreviation: MDCK, Madin-Darby canine kidney.

${ }^{a} \mathrm{~A}$ total of $13 \%$ of sequenced viruses belonged to the 3C.3a genetic group, but all were isolated in April 2018, well after the influenza A(H3N2) cases included in serologic analyses were identified.

${ }^{b}$ Neuraminidase-inhibition assay target.

enzyme-linked lectin assays). Titers below the limits of detection for each assay (ie, $<10)$ were assigned a value of half the threshold value (ie, 5).

\section{Sequencing, Phylogenetics Analysis, and Structure Analysis}

To characterize the viruses circulating in our source population during the study period, we identified all 29 influenza A(H3N2) virus-positive specimens with a RT-PCR cycle threshold $<30$ from University of Michigan participants enrolled during the full surveillance period (27 November 2017-24 April 2018). Six of these 29 were included as cases in the serologic analyses described above; the remaining 9 cases had a RT-PCR cycle threshold $\geq 30$. Segment 4 was amplified from extracted viral RNA, using the SuperScript III One-Step RT-PCR Platinum Taq HiFi Kit (Invitrogen 12574) and primers HA_H3N2_F (5'-AGCAAAA GCAGGGGATAATT CTATTAACCATG-3') and HA_H3N2_R (5'-AGTAGAAACAA GGGTGTTTTTAAT TAATGCACTC- $\left.3^{\prime}\right)$. The thermocycler protocol was as follows: $50^{\circ} \mathrm{C}$ for 60 minutes; $94^{\circ} \mathrm{C}$ for 2 minutes; 30 cycles of $94^{\circ} \mathrm{C}$ for 30 seconds, $54^{\circ} \mathrm{C}$ for 30 seconds, and $68^{\circ} \mathrm{C}$ for 3 minutes; and a final 5 -minute extension at $68^{\circ} \mathrm{C}$. All RT-PCR products were purified prior to Sanger sequencing. Twentythree samples yielded sequence of sufficient quality for subsequent analysis.

Additional reference sequences representing circulating genetic groups were identified using publicly available sources [17] and downloaded from the Global Initiative on Sharing All Influenza Data and GenBank (for all strains and reference numbers, see Supplementary Table 1). Sequences were aligned using MUSCLE [18] with default parameters in MegAlign Pro, version 13.0.0 (Lasergene). The alignments were trimmed to the HA coding region, using Aliview, version 1.23 [19]. Nucleotide substitution model selection was performed using jmodeltest, version 2.1.10 [20]. Maximum likelihood phylogenetic trees were generated in RAxML, version 8 [21], with a general time-reversible $\Gamma$ model and 1000 bootstraps. Trees were visualized and edited using FigTree, version 1.4.2. HA structure was generated using the PyMOL (Molecular Graphics System, Schrödinger).

\section{Statistical Analysis}

Individual antibody titers were $\log _{2}$ transformed for analysis; original values were divided by half the threshold value of detection (ie, 5) to set the starting point of the log scale to 0 prior to transformation. Mean $\log _{2}$ titers were calculated and compared by case status and influenza vaccination status, using $t$ tests. The correlation of $\log _{2}$ titers against each virus were assessed using Spearman rank correlation coefficients $(\rho)$. The independent effects of strain-specific MN titers on protection from infection was estimated in logistic regression models, with RT-PCRconfirmed influenza $\mathrm{A}(\mathrm{H} 3 \mathrm{~N} 2)$ virus infection as the outcome and $\log _{2} \mathrm{MN}$ titers as continuous predictors. Specific MN titers included in this model included those measured against the egg-grown influenza A/Hong Kong/4801/2014(H3N2) vaccine strain and the influenza A/Washington/16/2017(H3N2) circulating strain most closely related to the viruses that circulated in the local area. Odds ratios obtained from the model are interpreted as the factor reduction in the odds of influenza $\mathrm{A}(\mathrm{H} 3 \mathrm{~N} 2)$ virus infection associated with a 2 -fold increase in MN titer against influenza A/Hong Kong/4801/2014(H3N2) virus, holding the influenza A/Washington/16/2017(H3N2) virus-specific antibody titer constant, or vice versa.

Statistical analyses were performed using SAS software (release 9.4; SAS Institute) and R (version 3.4.3; packages: ggplot2); a $P$ value of $<.05$ or a positive lower bound of a $95 \%$ confidence interval (CI) were considered to indicate statistical significance.

\section{RESULTS}

\section{Genetic Characterization of Circulating Viruses}

Influenza $A(\mathrm{H} 3 \mathrm{~N} 2)$ viruses from 23 hospitalized cases with a RT-PCR cycle threshold $<30$ were successfully sequenced to determine the characteristics of the strains circulating locally. Analyses determined that 19 viruses (83\%) belonged to the 3C.2a2 genetic group similar to influenza A/ Washington/16/2017 virus (Figure 2). A single virus belonged to the 3C.2a1a group similar to influenza $\mathrm{A} /$ Singapore/ Infimh-16-0019/2016 virus. Three viruses (13\%) belonging to the 3C.3a genetic group were also identified, all of which 


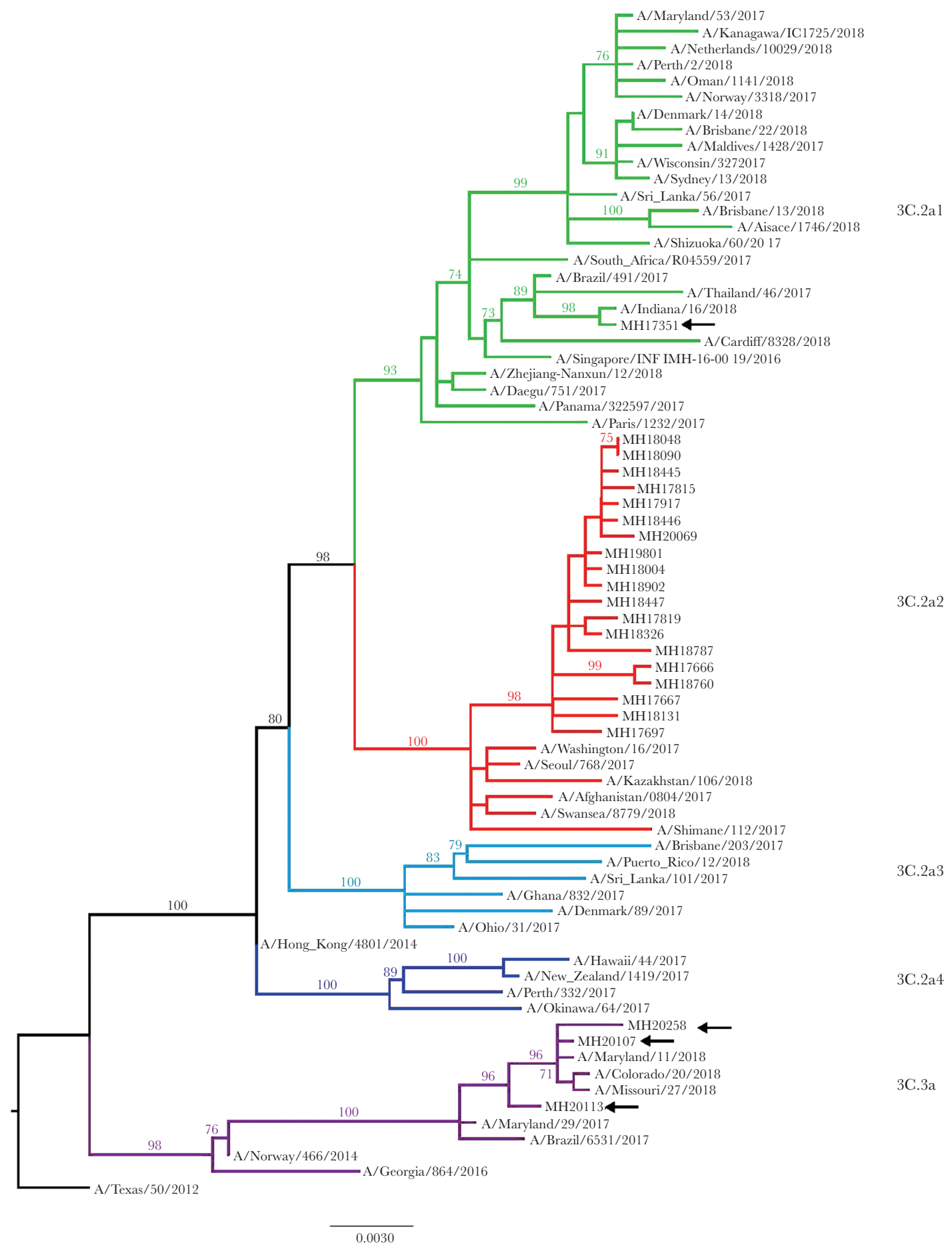

Figure 2. Maximum likelihood trees of hemagglutinin (HA) sequences, by clade. Branches of maximum likelihood trees of HA sequences are color-coded by clade (3C.2a13C.2a4 and 3C.3a; see notation near tips). The outgroup is influenza A/Texas/50/2012 virus. Samples from the Hospitalized Adult Influenza Vaccine Effectiveness Network cohort begin with "MH"; see clade 3C.2a2 $(n=19)$ and 4 additional sequences (black arrows). Clade assignments are based on locations of the reference sequences in nextstrain.org. Bootstrap values $\geq 70$ are shown.

were isolated in April 2018. Viruses descendent from the 3C.2a genetic group and serum specimens collected during the first half of the influenza outbreak were used in serological analysis (Table 1). Compared with wild-type SIAT1-grown influenza A/Washington/16/2017 virus and SIAT1-grown influenza A/Hong Kong/4801/2014 virus, vaccine-like eggpropagated influenza A/Hong Kong/4801/2014 virus has eggadapted changes at $\mathrm{T} 160 \mathrm{~K}$ (antigenic site $\mathrm{B}$, causing a loss of glycosylation motif), L194P (antigenic site B), and N96S (antigenic site D; Figure 3). 


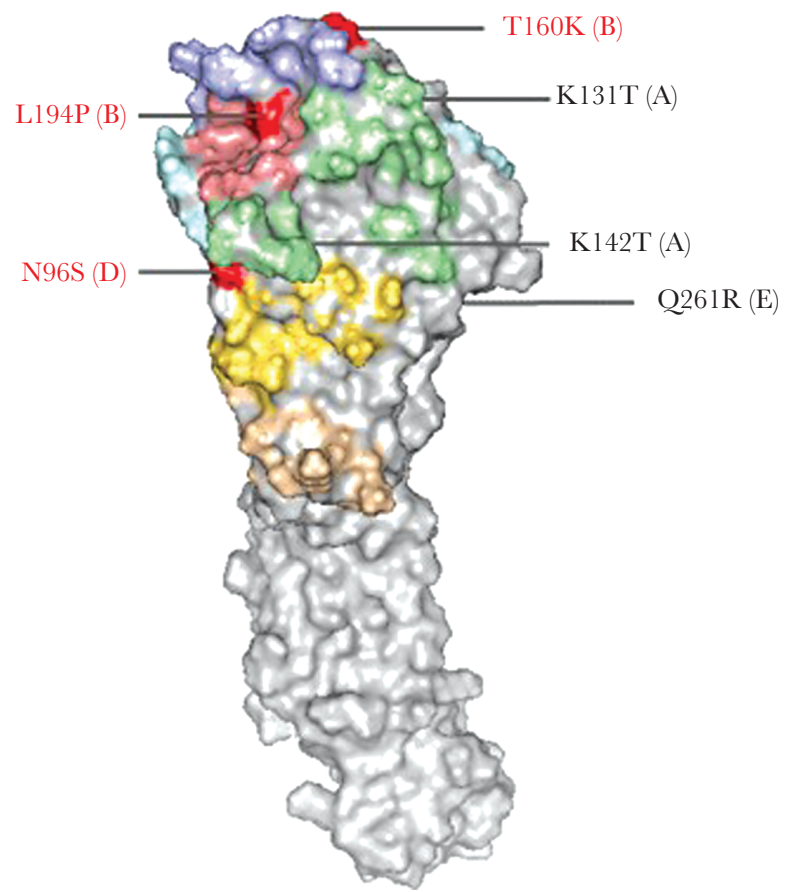

Figure 3. Differences in influenza $\mathrm{A}(\mathrm{H} 3 \mathrm{~N} 2)$ virus hemagglutinins (HAs) between egg-propagated influenza A/Hong Kong/4801/2014 virus and Madin-Darby canine kidney (MDCK)-SIAT1 cell-propagated influenza A/Washington/16/2017 virus. Amino acid changes highlighted in red on the HA structure are those associated with egg adaptation in egg-grown influenza A/Hong Kong/4801/2014 virus. Potential antigenic sites are indicated in parenthesis.

\section{Serologic Studies in Influenza A(H3N2) Virus-Infected Cases and Uninfected Controls}

Serologic analyses were performed on specimens collected from the subset of participants enrolled between 27 November 2017 and 31 January 2018, including all 15 influenza A(H3N2) virus-infected cases identified by that date and 15 uninfected controls. Participants ranged in age from 20 to 93 years (median, 37 years), 53\% were women, $63 \%$ were white, $60 \%$ received an influenza vaccine, $38 \%$ reported fair or poor general health, and $41 \%$ were frail. Cases and controls were comparable in terms of age, sex, race, and date of enrollment (Table 2). Control subjects were more likely to report fair or poor general health, were more likely to be frail, and were more likely to be vaccinated; however, these differences were not statistically significant. Serum specimens were collected $<5$ days after illness onset from $73 \%$ of cases and controls, combined.

Both neutralizing and NAI antibody titers to various influenza virus antigens in cases and controls combined were first determined. Overall titers measured by $\mathrm{MN}$ assays were highest against the egg-grown influenza A/HongKong/4801/2014 3C.2a vaccine strain (geometric mean titer [GMT], 97; 95\% CI, 53-177). Titers against cell-grown strains were lower than against the egg-grown strain, and titers were comparable between the cell-grown influenza A/HongKong/4801/2014 vaccine strain and the following 4 influenza $\mathrm{A}(\mathrm{H} 3 \mathrm{~N} 2)$ strains representative of those that circulated: influenza A/Washington/16/2017 strain 3C.2a2, influenza A/Singapore/Infimh-16-0019/2016 strain 3C.2a1, influenza A/Maryland/53/2017 strain 3C.2a1b+135N, and influenza A/Wisconsin/327/2017 strain 3C.2a1b $+135 \mathrm{~K}$ (GMT range, 19-34). NAI GMTs were $>40$ against both influenza A/HongKong/4801/2014 virus (GMT, 54; 95\% CI, 33-89) and influenza A/Singapore/Infimh-16-0019/2016 virus (GMT, 41; 95\% CI, 24-69; Supplementary Table 2).

The correlation of titers against egg- and cell-grown viruses in cases and controls combined was next evaluated. Antibody titers against egg-grown influenza A/HongKong/4801/2014 virus were most strongly correlated with titers against cellgrown influenza A/HongKong/4801/2014 virus titers $(\rho=0.71)$ and titers against influenza A/Wisconsin/327/2017 virus $(\rho=0.70)$ and less correlated with antibody titers to influenza A/ Singapore/Infimh-16-0019/2016 virus $(\rho=0.56)$ and influenza A/Washington/16/2017 virus ( $\rho=0.53$; Supplementary Figure $1)$. Correlations between antibody titers against each of the cellgrown viruses were higher ( $\rho$ range, $0.80-0.90$ ) to each other than to the egg-grown influenza A/HongKong/4801/2014 strain.

\section{Relation of Antibody Titers to Vaccination Status}

There were 18 individuals who had been vaccinated, and the specific influenza vaccine they received could be confirmed for 15 . All 15 individuals with confirmed vaccine product information received vaccines containing inactivated egg-grown virus; 10 received quadrivalent standard-dose vaccines (9 vaccines were produced by GSK, and 1 was produced by Seqirus), 3 received trivalent high dose vaccines (produced by Sanofi Pasteur), 1 received trivalent adjuvanted vaccine (produced by Seqirus), and 1 received trivalent unadjuvanted vaccine (produced by Seqirus). Vaccinated individuals had significantly higher titers against the egg-grown influenza A/HongKong/4801/2014 vaccine strain than unvaccinated individuals (GMT, 173 vs $41 ; P=.01$; Table 3 and Supplementary Figure $2 B$ ). For all other viruses, including the cell-grown influenza A/HongKong/4801/2014 vaccine strain, $\mathrm{MN}$ titers were similar between unvaccinated and vaccinated individuals (Table 3 and Supplementary Figure $2 B$ ). Interestingly, NAI titers were significantly higher for vaccinated individuals, compared with those for unvaccinated individuals (Table 3 and Supplementary Figure $3 B$ ) for both the influenza A/HongKong/4801/2014 strain (GMT, 80 vs 30; $P=.05$ ) and the influenza A/Singapore/Infimh-16-0019/2016 strain (GMT, 63 vs $21 ; P=.03)$.

\section{Relation of Antibody Titers to Infection Status}

Eight of 15 individuals (53\%) with influenza virus infection had been vaccinated against influenza in the 2017-2018 season. The majority of these vaccine failures had low MN titers against influenza A/Washington/16/2017 virus, which is most similar to those $2 \mathrm{a} 2$ viruses that predominated in the region. For all target strains, uninfected controls had higher $\mathrm{MN}$ titers than influenza A(H3N2) virus-infected cases (Table 3 and Supplementary Figure 
Table 2. Characteristics of Hospitalized Influenza A(H3N2) Virus-Infected Cases and Uninfected Controls

\begin{tabular}{|c|c|c|c|}
\hline Characteristic & Cases $(n=15)$ & $\begin{array}{l}\text { Controls } \\
(n=15)\end{array}$ & $P$ \\
\hline Age, $y^{a}$ & $34(22-63)$ & $37(28-62)$ & .62 \\
\hline Sex & & & .46 \\
\hline Female & $9(60)$ & $7(47)$ & \\
\hline Male & $6(40)$ & $8(53)$ & \\
\hline Race/ethnicity & & & .70 \\
\hline White (not Hispanic) & $10(67)$ & $9(60)$ & \\
\hline Other & $5(33)$ & $6(40)$ & \\
\hline Reported general health status & & & .32 \\
\hline Excellent/very good/good & $10(71)$ & $8(53)$ & \\
\hline Fair/poor & $4(29)$ & $7(47)$ & \\
\hline Frailty status & & & .18 \\
\hline Not frail & $10(71)$ & $7(47)$ & \\
\hline Frail & $4(29)$ & $8(53)$ & \\
\hline $\begin{array}{l}\text { Time from illness onset to } \\
\text { blood specimen collection, } d\end{array}$ & & & 1.00 \\
\hline $0-4$ & $11(73)$ & $11(73)$ & \\
\hline $5-9$ & $4(27)$ & $4(27)$ & \\
\hline Influenza vaccination status & & & .14 \\
\hline Vaccinated & $7(47)$ & $11(73)$ & \\
\hline Unvaccinated & $8(53)$ & $4(27)$ & \\
\hline Date of enrollment & $\begin{array}{l}18 \text { Jan } 2018 \\
\text { (8-27 Jan } \\
2018)\end{array}$ & $\begin{array}{c}9 \text { Jan } 2018 \text { (19 } \\
\text { Dec 2017-22 } \\
\text { Jan 2018) }\end{array}$ & .12 \\
\hline
\end{tabular}

Data are median value (interquartile range) or no. (\%) of participants.

${ }^{a}$ Ages ranged from 20 to 93 years overall.

$2 A$ ). These differences between cases and controls were statistically significant for all cell-grown viruses representative of circulating viruses ( $P<.01$ for all comparisons), but the differences were only marginally significant for antibody titers against eggand cell-grown influenza $\mathrm{A} /$ HongKong/4801/2014 vaccine strains ( $P=.05$ and $P=.09$, respectively). In contrast, NAI titers were not substantially different between cases and controls for either neuraminidase target (Table 3 and Supplementary Figure $3 A$ ).

We used multivariable logistic regression models to determine whether the apparent association between antibody against egg-grown influenza A/HongKong/4801/2014 virus and a reduced odds of influenza $\mathrm{A}(\mathrm{H} 3 \mathrm{~N} 2)$ virus infection was independent of the reduction in infection associated with antibody against influenza A/Washington/16/2017 virus, which was most similar to viruses that predominated in this population (Supplementary Table 3). In unadjusted models, $\mathrm{MN}$ titers against egg-grown influenza $\mathrm{A} /$ HongKong/4801/2014 virus (odds ratio, 0.71; 95\% CI, .50-1.01) and influenza A/Washington/16/2017 virus (odds ratio, 0.40; 95\% CI, .19-.86) were both associated with a reduced odds of infection, but only the effect for influenza A/Washington/16/2017 virus was statistically significant. These effects correspond to an estimated $50 \%$ reduction in the odds of infection for an individual with a titer of 20.3 against egg-grown influenza A/HongKong/4801/2014 virus and a titer of 8.5 against influenza A/Washington/16/2017 virus relative to an individual with an undetectable titer for each virus, respectively (Figure $4 \mathrm{~A}$ and $4 \mathrm{~B}$ ). The effects of both $\mathrm{MN}$ titers were somewhat attenuated and no longer statistically significant as estimated in adjusted models. After adjustment for titers against influenza A/Washington/16/2017 virus, the titer required for a $50 \%$ reduction in odds of infection increased to 95.3 for egg-grown influenza A/HongKong/4801/2014 virus (Figure 4C). After adjustment for titers against egg-grown influenza A/ HongKong/4801/2014 virus, the titer required for a 50\% reduction in the odds of infection increased to 9.3 for influenza A/ Washington/16/2017 virus (Figure 4D).

\section{DISCUSSION}

Our analysis of specimens from adults hospitalized with influenza A(H3N2) virus infection during the 2017-2018 influenza season demonstrated an antibody response specific to eggadapted strains that was significantly higher among vaccinated individuals. Additionally, antibody titers against cell-grown influenza A/HongKong/4801/2014(H3N2) virus were similar to those in unvaccinated individuals. In our study sample, this suggests that the antibody responses to vaccination in this population are highly specific to the egg-adapted strain, with little cross-reactivity to the circulating virus. Overall, titers against

Table 3. Microneutralization (MN) and Neuraminidase-Inhibition (NAI) Antibody Titers by Influenza A(H3N2) Virus Infection Status and Influenza Vaccination Status

\begin{tabular}{|c|c|c|c|c|c|c|}
\hline Assay, Virus & Infected & Uninfected & $P^{a}$ & Vaccinated & Unvaccinated & $P^{a}$ \\
\hline \multicolumn{7}{|l|}{ MN assay } \\
\hline A/HongKong/4801/2014-egg & $55(25-121)$ & $172(70-421)$ & .05 & $173(87-342)$ & $41(15-113)$ & .01 \\
\hline A/HongKong/4801/2014 & $22(14-35)$ & $39(24-64)$ & .09 & $35(23-53)$ & $23(13-42)$ & .21 \\
\hline AMashington/16/2017 & $14(11-20)$ & $32(19-53)$ & .01 & $22(15-34)$ & $20(11-36)$ & .72 \\
\hline A/Singapore/Infimh-16-0019/2016 & $14(10-19)$ & $35(22-56)$ & .002 & $22(15-33)$ & $21(12-39)$ & .86 \\
\hline A/Maryland/53/2017 & $13(10-18)$ & $28(17-46)$ & .008 & $21(14-30)$ & $17(10-31)$ & .56 \\
\hline AMisconsin/327/2017 & 19 (13-28) & $59(34-104)$ & .001 & $41(25-66)$ & $25(12-51)$ & .22 \\
\hline \multicolumn{7}{|l|}{ NAl assay } \\
\hline A/HongKong/4801/2014 & $46(20-103)$ & $63(32-125)$ & .52 & $80(42-153)$ & $30(14-64)$ & .05 \\
\hline A/Singapore/Infimh-16-0019/2016 & $48(18-129)$ & $35(21-57)$ & .53 & $63(34-119)$ & $21(9-51)$ & .03 \\
\hline
\end{tabular}

Data are geometric mean titer ( $95 \%$ confidence interval).

${ }^{a}$ By the $t$ test, for comparison of geometric mean titers between infected and uninfected individuals or between vaccinated and unvaccinated individuals 
A

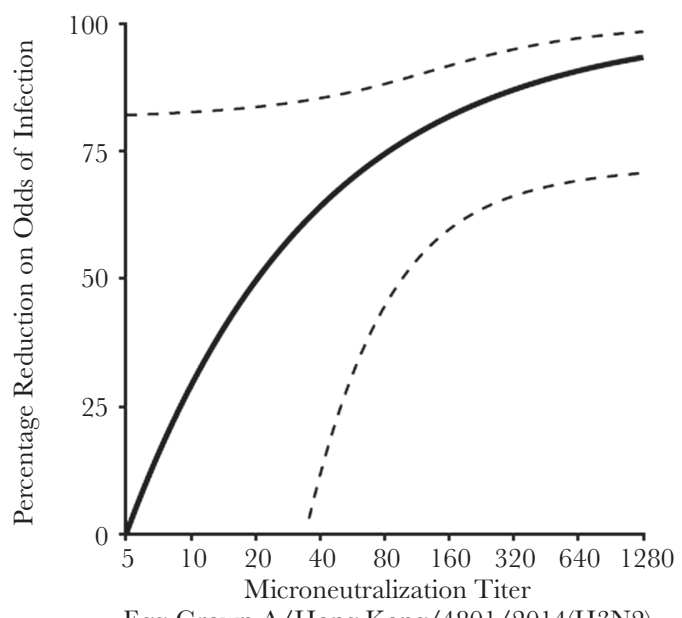

Egg-Grown A/Hong Kong/4801/2014(H3N2)

C

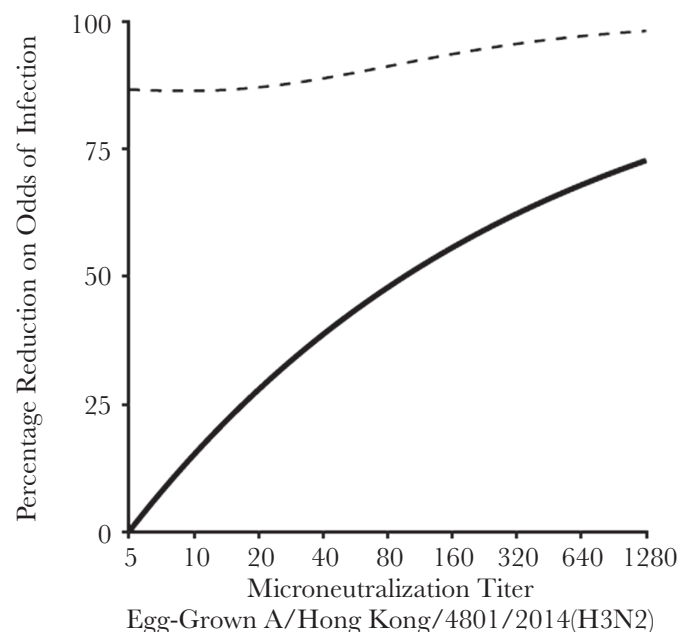

B

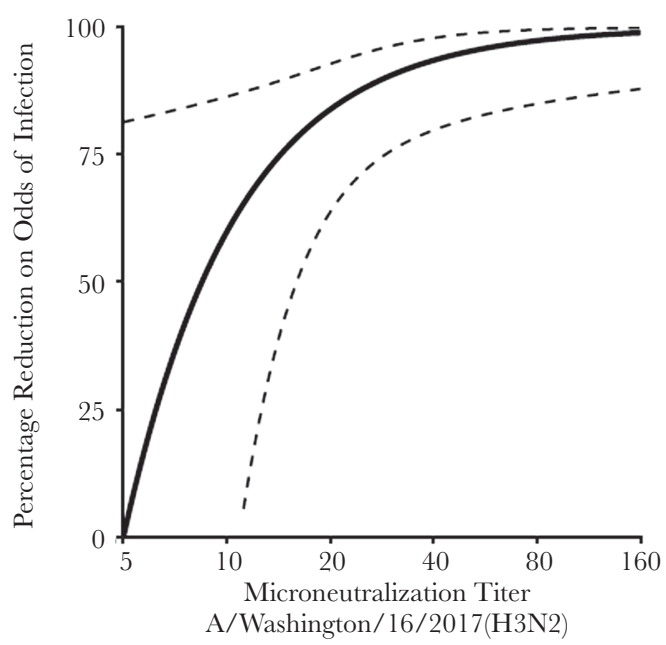

$\mathrm{D}$

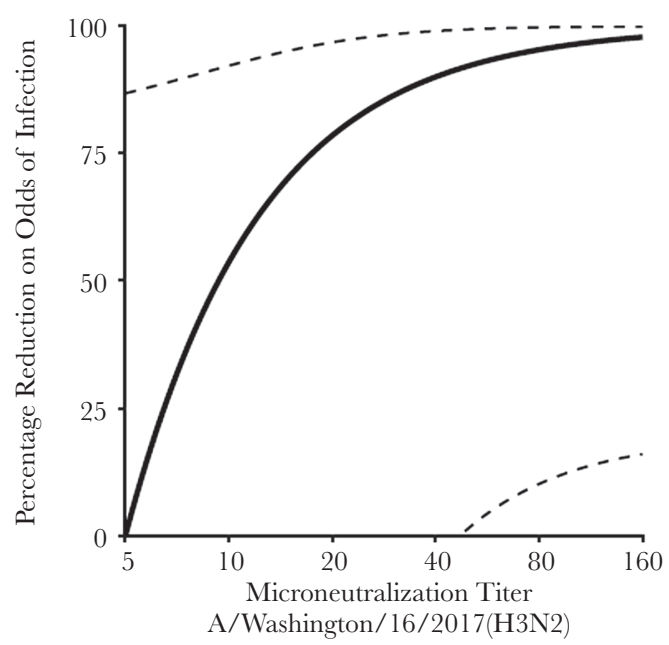

Figure 4. Estimated percentage reduction in the odds of influenza virus infection, by increasing microneutralization titers against egg-propagated influenza $A / H o n g$ Kong/4801/2014(H3N2) virus and influenza A/Washington/16/2017(H3N2) virus. $A$ and $B$, Unadjusted estimates for each virus target . C and D, Estimates adjusted for the titer against the other virus. Dotted lines indicate $95 \%$ confidence intervals.

wild-type influenza $\mathrm{A}(\mathrm{H} 3 \mathrm{~N} 2)$ strains 3C.2a1 and 3C.2a2 were significantly correlated with protection against infection during the 2017-2018 season. While titers against egg-adapted influenza A/Hong Kong/4801/2014(H3N2) virus were higher in the uninfected population, this was not statistically significant, and the magnitude of the correlation with protection was low.

Immunologic studies have proposed mechanisms for the lack of antibody cross-reactivity between egg-grown and circulating viruses. A study of plasmablasts in individuals receiving an $\mathrm{A} /$ California/07/2009(H1N1) vaccine strain found that individuals with a highly specific antibody response to a reversion present in the vaccine strain had no binding to the circulating influenza $\mathrm{A}(\mathrm{H} 1 \mathrm{~N} 1)$ strain [22]. With regard to more-recent influenza $\mathrm{A}(\mathrm{H} 3 \mathrm{~N} 2)$ strains, similar immunologic patterns of antibody misdirection have been reported for residue 160 in antigenic site B of HA [7]. Influenza A(H3N2) 3C.2a viruses that have circulated since the 2014-2015 season possess a K160T mutation that introduces a glycosylation to antigenic site B that was not present before 2014-2015. This mutation is reversed in the corresponding egg-propagated influenza virus 3C.2a egg-adapted strain present in the 2016-2017 and 2017-2018 vaccines, thus allowing the immune system to focus the antibody response on this epitope, which is otherwise shielded in circulating strains [7].

Previous studies of the role of existing antineuraminidase antibody titers as a correlate of protection provide conflicting evidence [10, 23-25]. In this study, antibody against the neuraminidase of influenza A/HongKong/4801/2014 virus and influenza A/Singapore/INFIMH-16-0019/2016 virus was not associated with protection against infection. Although neuraminidase content is not standardized in influenza vaccines, we observed that antineuraminidase antibody titers were higher among vaccinated individuals, suggesting that some of the existing antineuraminidase antibody in this population resulted from vaccination. This is consistent with previous studies 
that have directly observed a neuraminidase-directed antibody response following vaccination [26, 27]. Given questions about the quality of vaccine-induced neuraminidase antibody [28], it is clear that more work needs to be done to understand antigenic drift of the neuraminidase and how and when antineuraminidase antibodies mediate protection.

The serum specimens in this study were collected at a single time point early during acute illness. We have previously described evidence that antibody levels measured in acute specimens such as these reflects preinfection immune status, rather than a de novo response to infection [10]. However, although we demonstrated differences in antibody titer by vaccination status, we were unable to measure the magnitude of the antibody response to vaccination, owing to the lack of a paired prevaccination specimen. In addition, our conclusions are limited by the inclusion of a small number of specimens from a single hospital. Because the severity of the 2017-2018 epidemic was evident early in the season, we prioritized rapid serologic testing of the specimens that were available to date during the first half of the season. These 2 factors may limit the broad generalizability of the results; however, the influenza viruses that circulated locally overall and during the analysis period were similar to those that predominated nationwide during the same season. The small sample size also precluded our ability to perform additional subanalyses, to assess whether results might vary within specific age groups or by history of prior vaccination.

In one sense, the 2017-2018 influenza vaccine appears to have worked as intended: vaccinated individuals had high antibody titers against the influenza virus strain included in the vaccine. Our study adds to the evidence that egg adaptations in the 2017-2018 influenza vaccine strain elicited antibodies to epitopes not conserved in circulating strains. The degree to which this contributed to the marked severity of the 2017-2018 season remains to be determined. Several vaccine products have been introduced into the US vaccine market that are produced in cells and do not have the same emergent egg adaptations seen in recent influenza $\mathrm{A}(\mathrm{H} 3 \mathrm{~N} 2)$ vaccine strains. At this time, evidence for the effectiveness of these vaccines relative to those produced in eggs is limited because few head-to-head trials have been performed. Early data suggest that cell-based vaccines have somewhat improved protection in clinical trials $[29,30]$ and in the community [31]. Unfortunately, these vaccines are still dependent on selecting viruses that are close antigenic matches to those that will circulate and are subject to the limitations of annual strain selection time lines. This underscores the need for the development of the next-generation of influenza vaccines that provide better, broader, and longer-lasting protection [32].

\section{Supplementary Data}

Supplementary materials are available at The Journal of Infectious Diseases online. Consisting of data provided by the authors to benefit the reader, the posted materials are not copyedited and are the sole responsibility of the authors, so questions or comments should be addressed to the corresponding author.

\section{Notes}

Acknowledgments. We thank Maryna Eichelberger, Hongquan Wan, Jin Gao, and Laura Couzens (Food and Drug Administration), for technical support and providing reassortant influenza viruses for use in the enzyme-linked lectin assays; St Jude Children's Research Hospital, for providing plasmids that were used to generate these reassortant influenza viruses; and Mrs F. Liaini Gross, Lauren Horner, and Makeda Kay (Influenza Division, Centers for Disease Control and Prevention), for technical support for virus propagation and specimen management.

Disclaimer. The findings and conclusions in this report are those of the authors and do not necessarily represent the views of the Centers for Disease Control and Prevention.

Financial support. This work was supported by the Centers for Disease Control and Prevention (U01 IP000974) and the National Institutes of Health National Center for Advancing Translational Sciences (UL1TR002240).

Potential conflicts of interest. E. T. M has received grant support from Merck and Pfizer for work unrelated to this report. A. S. M. has received grant support from Sanofi Pasteur and consultancy fees from Sanofi, GSK, and Novavax for work unrelated to this report. A. S. L. has received consultancy fees from Sanofi for work unrelated to this report. J.M.F. has received nonfinancial support from Sanofi Pasteur for work unrelated to this report. All other authors reported no potential conflicts.

All authors have submitted the ICMJE Form for Disclosure of Potential Conflicts of Interest. Conflicts that the editors consider relevant to the content of the manuscript have been disclosed.

\section{References}

1. Garten R, Blanton L, Eala AIA, et al. Update: influenza activity in the United States during the 2017-18 season and composition of the 2018-19 influenza vaccine. MMWR Morb Mortal Wkly Rep 2018; 67:634-42.

2. World Health Organization. Recommended composition of influenza virus vaccines for use in the 2018-2019 northern hemisphere influenza season. 2018. http://www.who.int/ influenza/vaccines/virus/recommendations/2018_19_ north/en/. Accessed 20 June 2018.

3. Flannery B, Zimmerman RK, Gubareva LV, et al. Enhanced genetic characterization of influenza $\mathrm{A}(\mathrm{H} 3 \mathrm{~N} 2)$ viruses and vaccine effectiveness by genetic group, 2014-2015. J Infect Dis 2016; 214:1010-9.

4. Belongia EA, Simpson MD, King JP, et al. Variable influenza vaccine effectiveness by subtype: a systematic review and 
meta-analysis of test-negative design studies. Lancet Infect Dis 2016; 16:942-51.

5. Skowronski DM, Janjua NZ, De Serres G, et al. Low 2012-13 influenza vaccine effectiveness associated with mutation in the egg-adapted $\mathrm{H} 3 \mathrm{~N} 2$ vaccine strain not antigenic drift in circulating viruses. PLoS One 2014; 9. doi: 10.1371/journal. pone.0092153.

6. Wu NC, Zost SJ, Thompson AJ, et al. A structural explanation for the low effectiveness of the seasonal influenza H3N2 vaccine. PLoS Pathog 2017; 13:e1006682.

7. Zost SJ, Parkhouse K, Gumina ME, et al. Contemporary $\mathrm{H} 3 \mathrm{~N} 2$ influenza viruses have a glycosylation site that alters binding of antibodies elicited by egg-adapted vaccine strains. Proc Natl Acad Sci U S A 2017; 114:12578-83.

8. Chambers BS, Parkhouse K, Ross TM, Alby K, Hensley SE. Identification of hemagglutinin residues responsible for H3N2 antigenic drift during the 2014-2015 influenza season. Cell Rep 2015; 12:1-6.

9. Wu NC, Zost SJ, Thompson AJ, et al. A structural explanation for the low effectiveness of the seasonal influenza H3N2 vaccine. PLoS Pathog 2017; 13:e1006682.

10. Petrie JG, Martin ET, Truscon R, et al. Evaluation of correlates of protection against influenza $\mathrm{A}(\mathrm{H} 3 \mathrm{~N} 2)$ and A(H1N1)pdm09 infection: Applications to the hospitalized patient population. Vaccine 2019; 37:1284-92.

11. Ferdinands JM, Gaglani M, Martin ET, et al. Prevention of influenza hospitalization among adults in the US, 2015-16: results from the US Hospitalized Adult Influenza Vaccine Effectiveness Network (HAIVEN). J Infect Dis 2018. doi:10.1093/infdis/jiy723.

12. Fried LP, Tangen CM, Walston J, et al.; Cardiovascular Health Study Collaborative Research Group. Frailty in older adults: evidence for a phenotype. J Gerontol A Biol Sci Med Sci 2001; 56:M146-56.

13. Petrie JG, Ohmit SE, Cheng CK, et al. Influenza vaccine effectiveness against antigenically drifted influenza higher than expected in hospitalized adults: 2014-2015. Clin Infect Dis 2016; 63:1017-25.

14. Gross FL, Bai Y, Jefferson S, Holiday C, Levine MZ. Measuring influenza neutralizing antibody responses to $\mathrm{A}(\mathrm{H} 3 \mathrm{~N} 2)$ viruses in human sera by microneutralization assays using MDCK-SIAT1 cells. J Vis Exp 2017. doi: $10.3791 / 56448$.

15. World Health Organization/World Organisation for Animal Health/Food and Agriculture Organization (WHO/OIE/ FAO) H5N1 Evolution Working Group. Revised and updated nomenclature for highly pathogenic avian influenza A (H5N1) viruses. Influenza Other Respir Viruses 2014; 8:384-8.

16. Couzens L, Gao J, Westgeest K, et al. An optimized enzymelinked lectin assay to measure influenza A virus neuraminidase inhibition antibody titers in human sera. J Virol Methods 2014; 210:7-14.
17. Neher RA, Bedford T. nextflu: real-time tracking of seasonal influenza virus evolution in humans. Bioinformatics 2015; 31:3546-8.

18. Edgar RC. MUSCLE: multiple sequence alignment with high accuracy and high throughput. Nucleic Acids Res 2004; 32:1792-7.

19. Larsson A. AliView: a fast and lightweight alignment viewer and editor for large datasets. Bioinformatics 2014; 30:3276-8.

20. Darriba D, Taboada GL, Doallo R, Posada D. jModelTest 2: more models, new heuristics and parallel computing. Nat Methods 2012; 9:772.

21. Stamatakis A. RAxML version 8: a tool for phylogenetic analysis and post-analysis of large phylogenies. Bioinformatics 2014; 30:1312-3.

22. Raymond DD, Stewart SM, Lee J, et al. Influenza immunization elicits antibodies specific for an egg-adapted vaccine strain. Nat Med 2016; 22:1465-9.

23. Monto AS, Petrie JG, Cross RT, et al. Antibody to influenza virus neuraminidase: an independent correlate of protection. J Infect Dis 2015; 212:1191-9.

24. Couch RB, Atmar RL, Franco LM, et al. Antibody correlates and predictors of immunity to naturally occurring influenza in humans and the importance of antibody to the neuraminidase. J Infect Dis 2013; 207:974-81.

25. Monto AS, Kendal AP. Effect of neuraminidase antibody on Hong Kong influenza. Lancet 1973; 1:623-5.

26. Monto AS, Petrie JG, Cross RT, et al. Antibody to influenza virus neuraminidase: an independent correlate of protection. J Infect Dis 2015; 212:1191-9.

27. Couch RB, Atmar RL, Keitel WA, et al. Randomized comparative study of the serum antihemagglutinin and antineuraminidase antibody responses to six licensed trivalent influenza vaccines. Vaccine 2012; 31:190-5.

28. Chen Y-Q, Wohlbold TJ, Zheng N-Y, et al. Influenza infection in humans induces broadly cross-reactive and protective neuraminidase-reactive antibodies. Cell 2018; 173:417-29.e10.

29. Dunkle LM, Izikson R, Patriarca P, et al.; PSC12 Study Team. Efficacy of recombinant influenza vaccine in adults 50 years of age or older. N Engl J Med 2017; 376:2427-36.

30. Frey S, Vesikari T, Szymczakiewicz-Multanowska A, et al. Clinical efficacy of cell culture-derived and egg-derived inactivated subunit influenza vaccines in healthy adults. Clin Infect Dis 2010; 51:997-1004.

31. Izurieta HS, Chillarige Y, Kelman J, et al. Relative effectiveness of cell-cultured and egg-based influenza vaccines among elderly persons in the United States, 2017-2018. J Infect Dis 2018. doi:10.1093/infdis/jiy716.

32. Erbelding EJ, Post DJ, Stemmy EJ, et al. A Universal Influenza Vaccine: The Strategic Plan for the National Institute of Allergy and Infectious Diseases. J Infect Dis 2018; 218:347-54. 